\title{
In Vivo Investigation of Cardiac Beneffits of Sodium Glucose Cotransporter Inhibition Using the Zebrafish Model
}

\author{
Asma M. Essa1, Noura J. Aldous'
}

Supervised by: Dr. Huseyin C. Yalcin ${ }^{2}$, Dr. Fatiha Benslimane ${ }^{2}$

${ }^{1}$ Biomedical Science Department, College of Health Sciences, Qatar University, Doha, Qatar

$$
\text { Biomedical Research Center, Qatar University, PO Box 2713, Doha, Qatar }
$$

\section{Abstract}

Type 2 diabetes mellitus (T2DM) affects $>16 \%$ of adults in Qatar. Newly emerging class of antidiabetic drugs focuses on SGLT inhibition were observed to reduce CVDs risks in diabetic patients. Up to date, the mechanism contributing to the $\mathrm{CV}$ benefits remains unrevealed. Zebrafish embryos were injected with different morpholinos to knockdown SGLT genes and study their effects on cardiac parameters. SGLT1 inhibition caused the most severe effects on zebrafish embryos with survival rate $\sim 10 \%$. It also caused tube-like structured heats with edema, affecting significantly the cardiac output and diameter, and increased cardiac markers expressions. Analysis acquired correlates with literature data of SGLT1 predominant expression in heart tissues.

\section{Introduction}

Until today, the exact mechanism of SGLT inhibition effects on CV tissues remains unclear. Therefore, this research aims to understand:

- Role of SGLT genes expression in normal individuals.

- Analyze the impact of SGLT1 and SGLT2 absence on the development of heart disease and function.

- Identify the relationship between SGLT1 and SGLT2 inhibition and compensatory effects.

\section{Methodology}

Zebrafish mating

$0.25 \mathrm{hpf}$ Morpholino injection

\section{hpf Tail flicking}

\section{Heart assessment}

RNA isolation and cDNA synthesis

\section{Gene expression using RT-qPCR}
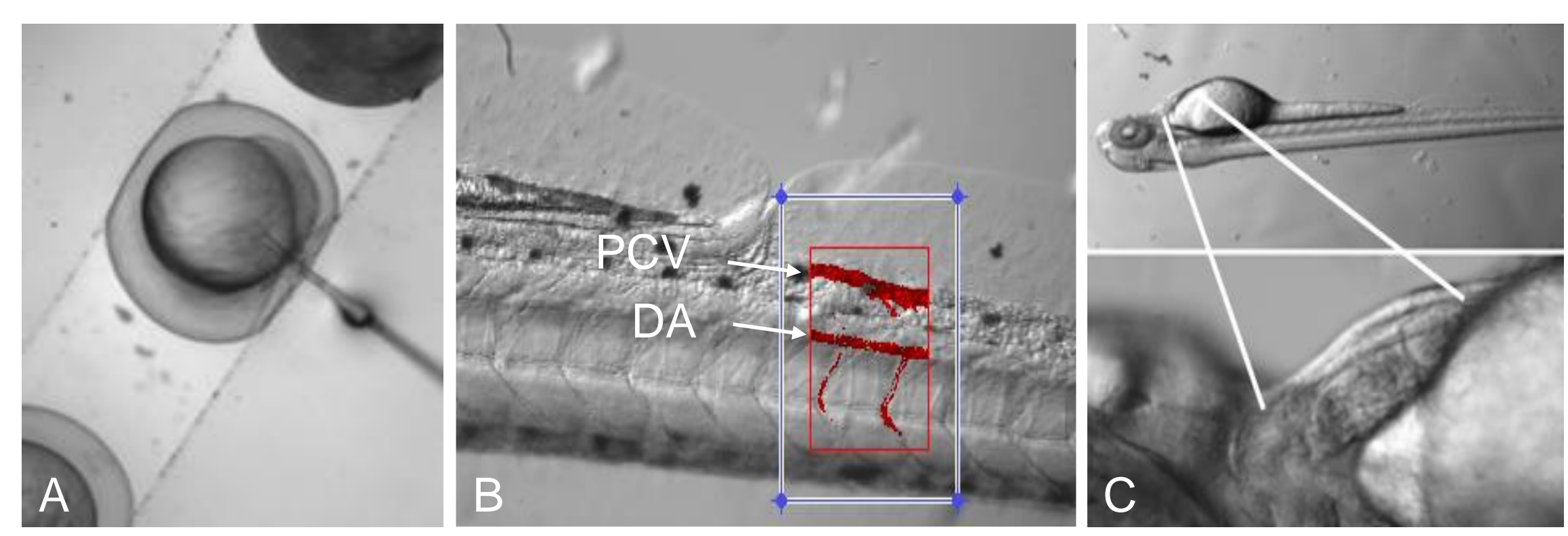

Figure 1. (A) MO injection process, (B) heart assessment using PCV and DA, (C) zebrafish heart location.

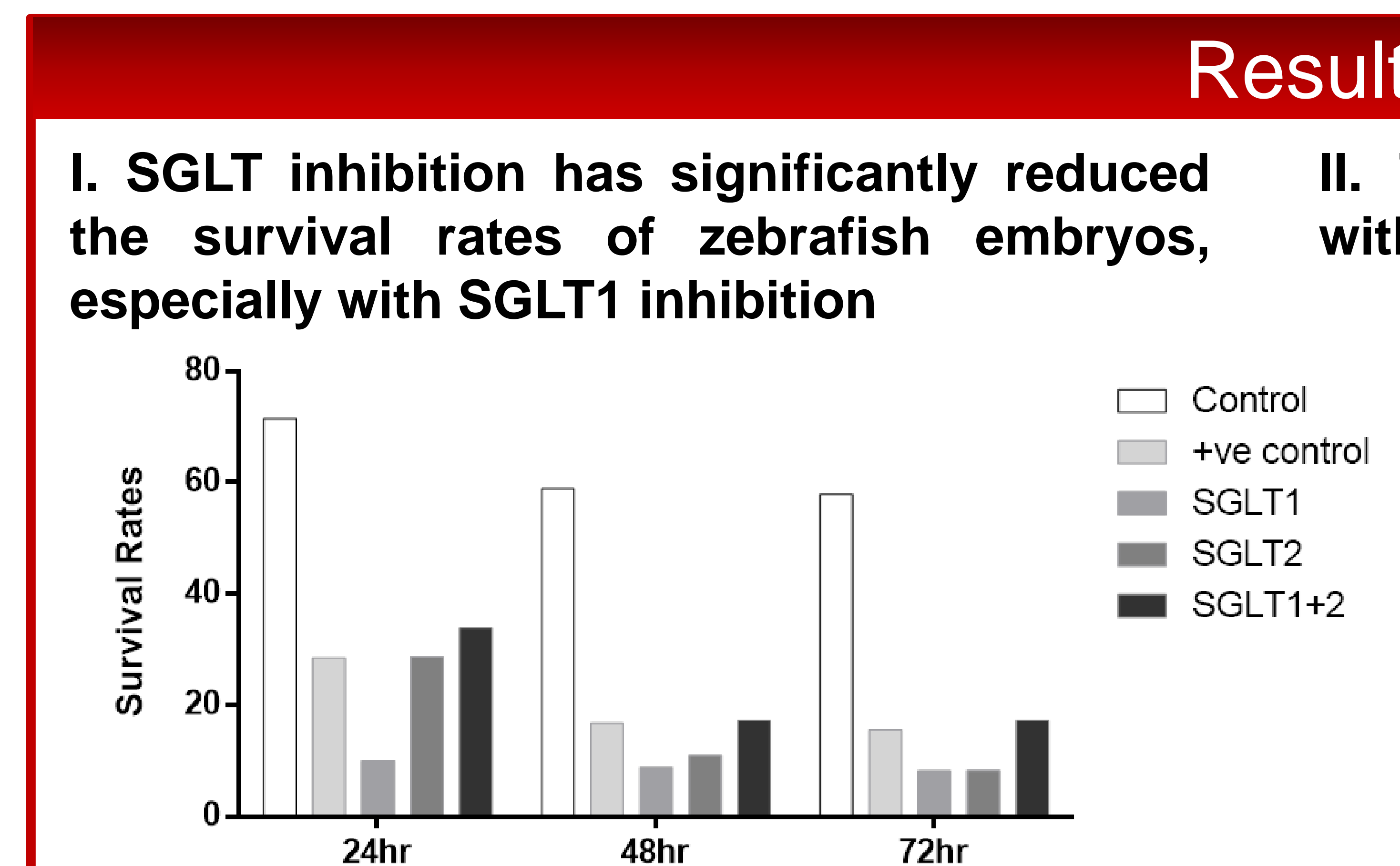

Figure 2. SGLT inhibition with MO has significantly affected the surviva rates of the embryos from the first $24 \mathrm{hpf}$, especially SGLT1 inhibition.

III. SGLT inhibition has affected heart structure and embryo development

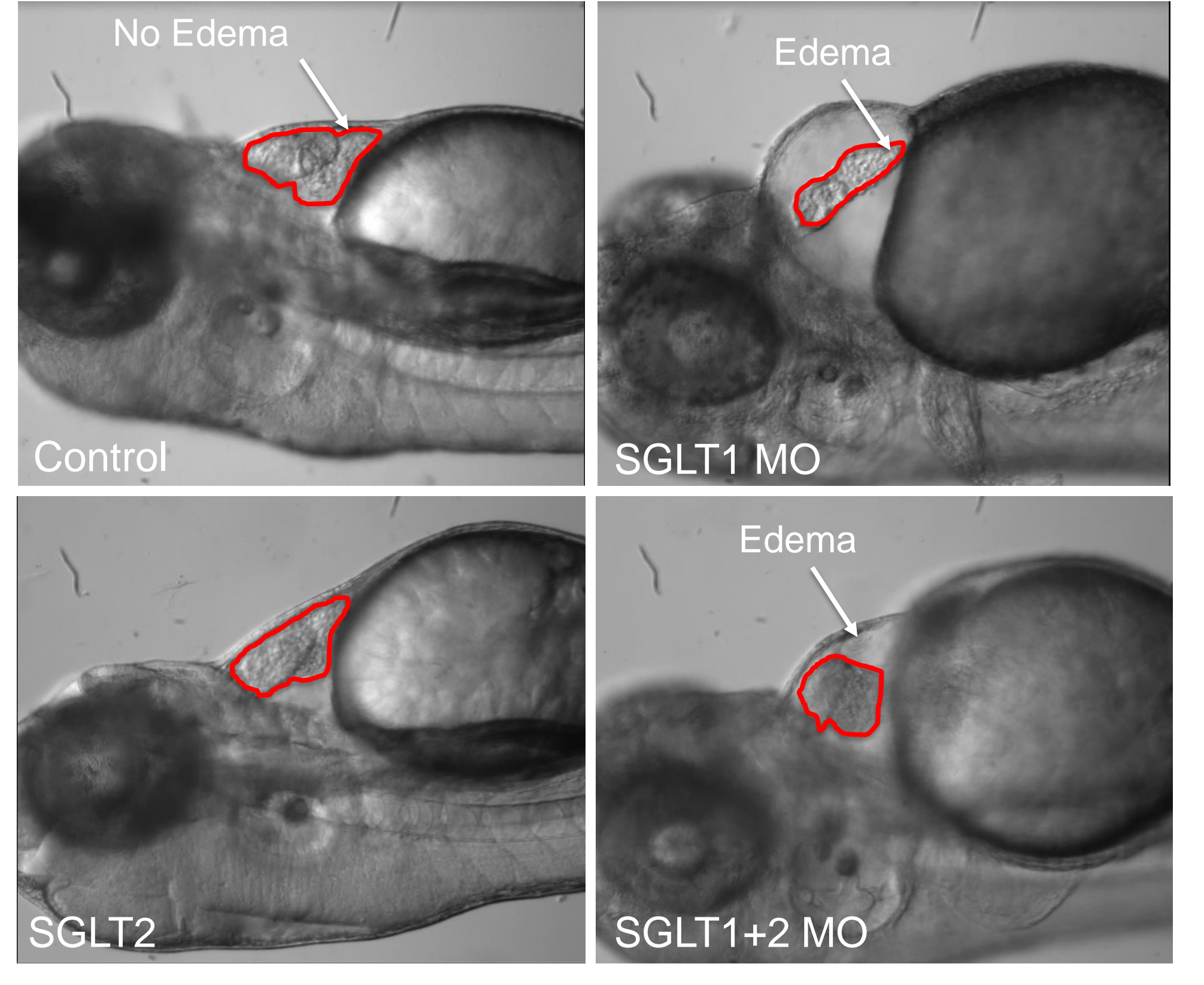

Figure 4. SGLT inhibition has significantly affected the heart structure in SGLT1 inhibition where it became tube-like with edema. SGLT2 inhibition resulted in a slightly elongated While SGLT1+2 resulted in slight edema with no visible chambers.

V. SGLT1 inhibition has significantly affected cardiac parameters
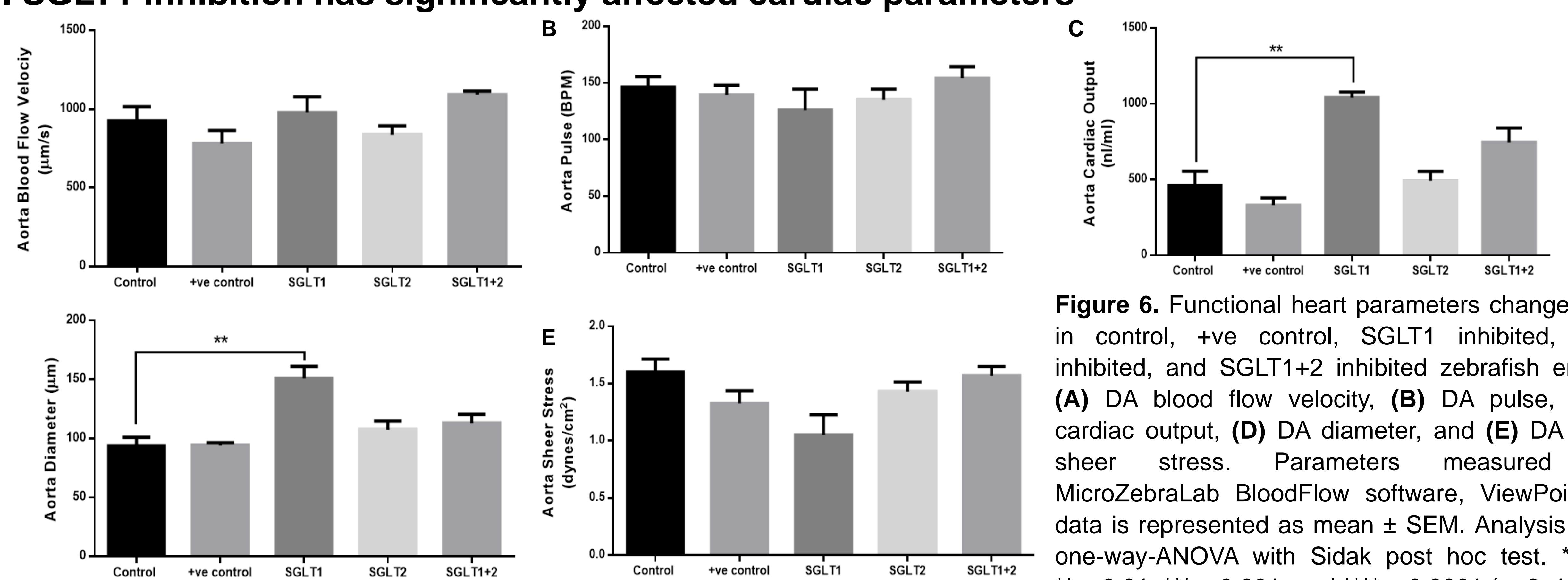

Figure 6. Functional heart parameters changes of DA control, +ve control, SGLT1 inhibited, SGLT2 (A) DA blood SGL1+2 inhibited zebrafish embryos. (A) DA blood flow velocity, (B) DA pulse, (C) DA (A) DA bucod cheer stress Parameters measured using MicroZebratab BloodFlow software, ViewPoint Al data is represented as mean \pm SEM. Analysis was by ne-way-ANOVA with Sidak post hoc test. ${ }^{*} p<0.05$ $p<0.01,{ }^{* * *} p<0.001$, and ${ }^{* * * *} p<0.0001(n=6 \pm 1)$.

V. SGLT1 inhibition has significantly increased cardiac markers expressions

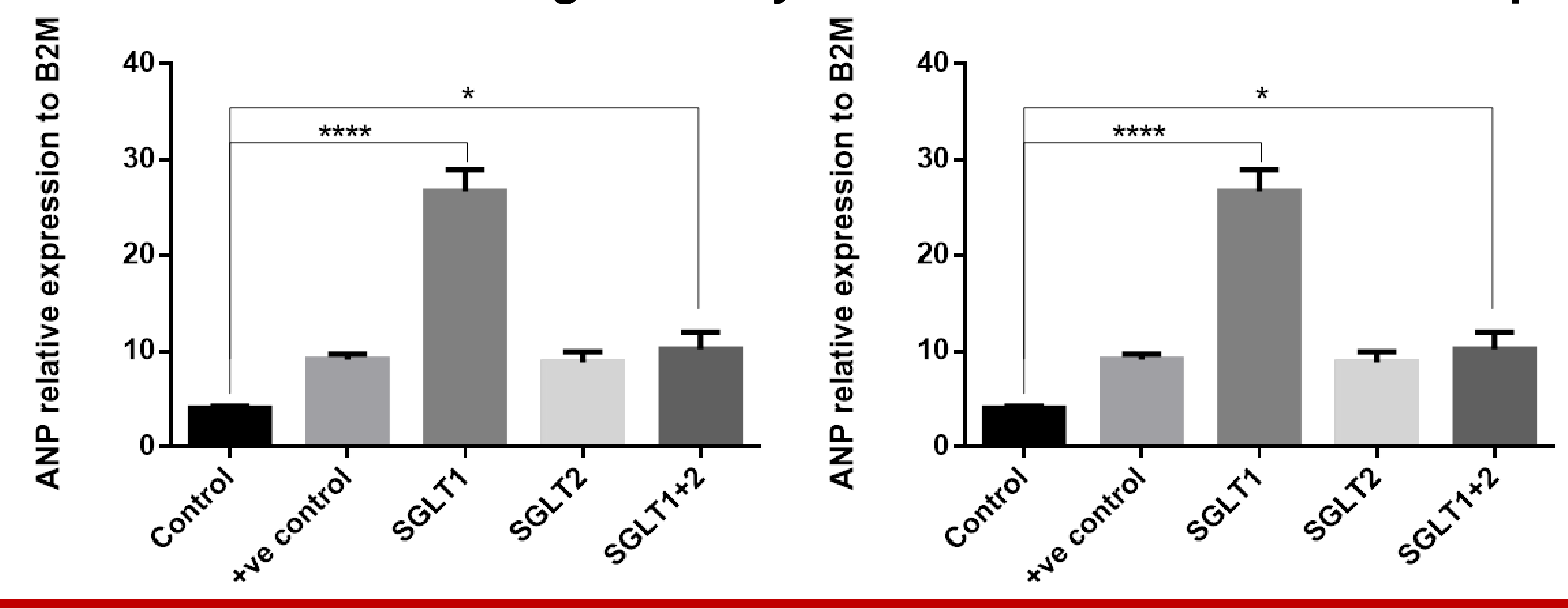

Figure 7. BNP and ANP relative gene expression to reference gene B2M in experimental groups. One-way ANOVA with Sidak post hoc test for multiple comparison. $\quad{ }^{*} \mathrm{p}<0.05, \quad{ }^{*} \mathrm{p}<0.01$ ${ }^{* * *} p<0.001$, and ${ }^{* * * *} p<0.0001(n=3)$.

\section{Conclusion}

- In comparison to wild type, SGLT1 knockdown triggered a delay in heart development, reduce vessel diameter and overall cardiac output. SGLT1 MO showed significant effects on structural and functional heart parameters with increased cardiac markers expressions that correlates with literature data of SGLT1 predominant expression in the heart.

- SGLT2 MO resulted in less severity on cardiac function since the expression is lower in heart tissues.

- SGLT1+2 MO has the least effect on cardiac parameters. We will increase the MO concentration to see if higher concentrations will result in phenotype without remarkable under-development of the embryos. 